\title{
Modalités de la pollinisation chez deux lignées de féverole de printemps (Vicia faba L var equina Steudel). Effets sur les coulures, la productivité et les taux de croisements
}

\author{
J Mesquida 1, J Le Guen 2, JN Tasei 3, S Carre 3, G Morin 2 \\ 1 INRA, centre de recherches de Rennes, laboratoire de zoologie; \\ 2 INRA, centre de recherches de Rennes, station d'amélioration des plantes, \\ domaine de la Motte au Vicomte, BP 29, 35650 Le Rheu; \\ ${ }^{3}$ INRA, centre de recherches de Poitou-Charentes, laboratoire de Zoologie, \\ F 86600 Lusignan, France
}

(Reçu le 16 février 1990; accepté le 22 juin 1990)

\begin{abstract}
Résumé - Les effets de différentes conditions de pollinisation : cages avec abeilles (Apis mellifera $L$ ); cages avec bourdons (Bombus lapidarius L); cages sans insectes; cages avec déclenchement manuel et extérieur sans cages, ont été étudiés sur les coulures des organes reproducteurs de 2 lignées de féverole : l'une qui s'autopollinise (D-27) et l'autre non (D-23). L'efficacité de la pollinisation a été vérifiée sur les nouaisons, les composantes de rendement et les taux de croisements. La pollinisation entomophile (abeilles ou bourdons) s'est montrée sans effets significatifs sur les coulures (62-68\% pour les coulures totales), les nouaisons (32-38\%) et les composantes du rendement (73,3-90,9 graines par pied et 31,6-39,9 g pour le rendement) de la lignée D-27. L'autopollinisation de cette lignée apparaît prépondérante. Chez la lignée D-23, au contraire, l'absence de pollinisateurs (abeilles ou bourdons) augmente significativement les coulures ( $86 \%$ contre $41 \%$ avec abeilles et $39 \%$ avec bourdons pour les coulures totales) et diminue significativement les nouaisons (14\% contre $59 \%$ avec abeilles et $61 \%$ avec bourdons) et les composantes du rendement (nombre de graines par pied : 8,6 contre 69 et 80 et rendement : 5,7 contre $31 \mathrm{~g}$ avec abeilles et $34 \mathrm{~g}$ avec bourdons). La pollinisation par l'intermédiaire d'abeilles ou de bourdons apparaît nécessaire et obligatoire chez ce génotype. Les taux de croisements de la lignée D-27 par la lignée D-23 ont été faibles, les valeurs obtenues dans les cages avec abeilles $(9,4 \%)$ et bourdons $(11,6 \%)$ ont été similaires mais supérieures à celles de l'extérieur $(5,1 \%)$.
\end{abstract}

Apis mellifera / Bombus / pollinisation / coulure / Vicia faba

\section{INTRODUCTION}

La féverole (Vicia faba L var equina Steudel) est une espèce autocompatible dont la biologie florale est intermédiaire entre l'auto- et l'allogamie (Berthelem, 1976).
Les variétés cultivées actuellement en France sont, pour certaines constituées de plantes qui s'autopollinisent très bien, mais pour la plupart représentées par un mélange de plantes à degré d'autopollinisation plus ou moins important. Pour ces 
dernières, le déclenchement de la colonne staminale est obligatoire et l'intervention d'insectes pollinisateurs est alors nécessaire. Dans ce cas, seule une faible partie des déclenchements peut être assurée par des chocs dus au vent.

La féverole est visitée par de nombreuses espèces d'insectes pollinisateurs efficaces dont les bourdons (Bombus spp) et les abeilles domestiques (Apis mellifera L). Les bourdons représentent environ $15 \%$ de la faune pollinisatrice de cette espèce végétale (Tasei, 1976) et les abeilles domestiques environ $80 \%$ (Free, 1962; Poulsen, 1973). Le rôle de la pollinisation entomophile et de ses effets sur la productivité de diverses variétés de féverole ont fait l'objet d'étude par Free (1970) et par Tasei (1984) en particulier.

Chez la féverole, le choix des critères de sélection pour le rendement est délicat en raison de l'instabilité de celui-ci dans le temps et dans l'espace et de l'importance des phénomènes de coulure qui se produisent entre l'initiation des fleurs et la maturité des gousses. Seule une faible proportion des fleurs formées donne des gousses récoltables (10-20\% selon Tasei, 1984). Ce phénomène de coulure s'observe d'ailleurs couramment chez d'autres Fabacées telles la luzerne (Medicago sativa L; Delaude et Tasei, 1972). Delaude (1973) relie ces phénomènes aux exigences de la plante vis-à-vis du milieu (climat, sol, fumure, eau, etc), ainsi qu'aux aspects physiologiques et génétiques de l'espèce. Ces coulures pourraient être aussi liées à des problèmes de pollinisation. Nous avons donc recherché les effets de différentes conditions de pollinisation sous cages et à l'extérieur sur la coulure des fleurs et des gousses de 2 lignées de féverole présentant une biologie florale différente : une lignée qui s'autopollinise et une lignée nécessitant le déclenchement de la colonne staminale.
Cette étude a également porté sur l'incidence des différentes modalités de pollinisation sur les composantes du rendement et les taux de croisements.

\section{MATÉRIELS ET MÉTHODES}

\section{Matériel végétal}

Les 2 lignées de féverole utilisées ont été fournies par la station d'amélioration des plantes de Dijon (DUC). Ce sont des lignées voisines génétiquement qui présentent les caractéristiques suivantes :

- Lignée D-27, qui s'autopollinise et à tégument de la graine beige-clair, (lignée homozygote récessive)

- Lignée D-23, à déclenchement obligatoire et à tégument de la graine violet (lignée homozygote dominante).

\section{Dispositif expérimental}

Les résultats présentés sont issus d'une expérimentation réalisée sur le domaine de I'INRA du Rheu (llle et Vilaine) en 1988.

Nous avons utilisé un dispositif en bloc randomisé avec 3 répétitions et 5 traitements. Les 5 traitements qui comportaient systématiquement les 2 variétés étaient les suivants : cage avec abeilles, cage avec bourdons, cage sans insectes en autopollinisation (ou contrôle), cage sans insectes avec déclenchement manuel et extérieur en pollinisation libre.

Du début de la floraison jusqu'à la récolte, les cages de $18 \mathrm{~m}^{3}(3 \times 3 \times 2 \mathrm{~m}, \mathrm{~L} \times \mathrm{I} \times \mathrm{h})$ étaient recouvertes d'un voile à mailles de $2 \mathrm{~mm}$ pour empêcher la pénétration d'insectes pollinisateurs. Chaque parcelle de $9 \mathrm{~m}^{2}$ comportait 2 lignes de 10 plantes de la lignée D-27 adjacentes à 2 lignes de 10 plantes de la lignée $D$ 23 avec un interligne de $0,5 \mathrm{~m}$ et $0,3 \mathrm{~m}$ entre les plantes. Le semis a été réalisé le 15 mars 1988.

Chaque cage avec abeilles comportait une colonie sur 3 cadres du type Dadant (5 000 abeilles environ) avec leur reine et du couvain. 
Les cages avec bourdons avaient reçu 2 reines de Bombus lapidarius $L$ en début de nidification dans leur caisse d'élevage du type préconisé par Pouvreau (1965). Dans chaque cage avec insectes, on a apporté en permanence dans des coupelles du sirop de sucre concentré ( $2 \mathrm{~kg}$ de sucre pour $1 \mathrm{l} / \mathrm{d}$ 'eau) et de l'eau. Les déclenchements manuels ont été réalisés sur toutes les fleurs au fur et à mesure de leur épanouissement en tirant délicatement sur la carène et l'étendard («tripping"), ce qui met en contact le pistil et le pollen.

\section{Observations}

Les observations ont porté sur :

\section{Les sécrétions nectarifères}

Les quantités de nectar sécrété par des fleurs de même âge sur 5 plantes par lignée ont été mesurées après pipetage à l'aide de micropipettes de $5 \mu$ l selon la méthode et la technique utilisées pour le colza (Mesquida et al, 1988).

\section{L'activité de butinage}

L'intensité de butinage des lignées a été mesurée par comptage sur $3 \mathrm{~min}$, du nombre d'abeilles et de bourdons venant butiner les fleurs de chaque lignée dans les cages ou les parcelles extérieures. Ce comptage a été répété 5 fois à intervalles de $20 \mathrm{~min}$. Le comportement de butinage a été déterminé par comptage du nombre de passages des abeilles différentes et des bourdons différents entre plantes d'une même lignée (passage «intra») ou entre plantes des lignées différentes (passages «inter»).

\section{Effets de la pollinisation sur les coulures}

Les comptages de noeuds reproducteurs, fleurs, jeunes gousses, gousses âgées et gousses mûres ont été réalisés systématiquement tous les 2 j sur 150 plantes ( 5 plantes par lignée et par traitement dans chaque bloc). Ces plantes ont été prises au hasard et numérotées. On a appelé «jeunes gousses» des gousses dont la taille était inférieure ou égale à $2 \mathrm{~cm}$, "gousses âgées" des gousses de taille supérieure à $2 \mathrm{~cm}$ et inférieure ou égale à $4 \mathrm{~cm}$ et "gousses mûres" celles qui ont atteint la maturité et qui sont récoltées. Ces comptages ont été réalisés sur un minimum de 15 noeuds reproducteurs consécutifs par plante. Pour simplifier, on les a regroupés au niveau de chaque plante en $3 \mathrm{sec}-$ teurs de 5 noeuds chacun (secteur 1 pour les noeuds 1-5; secteur 2 pour les noeuds 6-10 et secteur 3 pour les noeuds 11-15). Les pourcentages de coulure ont été calculés selon :

- taux de coulure des noeuds reproducteurs : $100 \times[(N r F L-N r G M) / N r F L]$;

- taux de coulure des fleurs :

$100-[(J G / F L) \times 100]$;

- taux de coulure des jeunes gousses :

100 - [(GA/JG) $\times 100]$;

- taux de coulure des gousses mûres :

$100-[(G M / G A) \times 100]$;

- taux de coulure des gousses totales :

$100-[(G M / J G) \times 100] ;$

- taux de coulure totale : $100-[(G M / F L) \times 100]$;

où $[(G M / F L) \times 100]$ correspond au taux de nouaison, avec

$N r F L=$ nombre de noeuds reproducteurs pendant la floraison;

NrGM = nombre de noeuds reproducteurs au stade «gousses mûres";

$F L=$ nombre de fleurs;

$J G=$ nombre de jeunes gousses;

$G A=$ nombre de gousses âgées;

$G M=$ nombre de gousses mûres récoltées .

\section{Effets de la pollinisation sur les composantes du rendement}

Les composantes du rendement ont porté sur le nombre de graines par gousse, le poids de 1000 graines, le poids de graines produites par plante et le nombre de graines produites par plante.

\section{Effets de la pollinisation sur les taux de croisements}

Les taux de croisements ont été calculés sur les descendances des plantes des divers traite- 
ments avec insectes. La coloration du tégument a été utilisée comme marqueur de croisement à partir de comptages du nombre de grains à téguments violets dans les descendances de la lignée D-27 à grains clairs. Les comptages ont été réalisés sur un échantillon de 300 plantes avec 3 répétitions.

\section{Analyse des données}

L'analyse des données a été réalisée sur la valeur moyenne des 5 plantes par répétition et non pas sur les valeurs des plantes individuelles. Dans ces conditions, l'analyse statistique des données a été réalisée après transformation des pourcentages (Arcsinus $\sqrt{ } P$ ) par une analyse de la variance à 3 facteurs étudiés suivie dans le cas de rejet de l'hypothèse nulle d'un test de comparaison de moyennes de Duncan. Les différences d'intensité de butinage des abeilles et des bourdons sur les lignées D-27 et D-23 et les différences du nombre de passages intra et interlignées, ont été interprétées au moyen du test $\chi^{2}$.

Les données rapportées sur les graphiques correspondent aux moyennes accompagnées de leur intervalle de confiance suivant la méthode proposée par Dagnélie (1973).

\section{RÉSULTATS}

\section{Sécrétions nectarifères}

La lignée D-27 a sécrété un nectar significativement $(P>0,02)$ plus abondant que la lignée D-23 $(0,56 \mu$ l contre $0,14 \mu$, fleur/ $24 \mathrm{~h}$ pour $\mathrm{D}-23$ ) et plus concentré en sucres totaux (30\% contre $19 \%)$.

\section{Activité de butinage des pollinisateurs}

L'activité de butinage des pollinisateurs a été satisfaisante pendant pratiquement toute la longue période de floraison des fé- veroles. A l'intérieur des cages avec abeilles, les densités de butineuses ont été nettement plus élevées que celles de l'extérieur (environ 20 butineuses $/ \mathrm{m}^{2}$ sous cages contre 0,5-2 à l'extérieur, au moment du maximum d'activité). La lignée $D$ 27 , plus nectarifère et plus florifère $39 \%$ de fleurs en plus : 9,82 fleurs par nceud contre 7,08 pour D-23), ne s'est cependant pas montrée significativement plus attractive que D-23 vis-à-vis des abeilles et des bourdons (pourcentages de visites : $46 \%$ sur D-27 contre $54 \%$ sur D-23 pour les abeilles, $\chi^{2}=3,17$ à $3 \mathrm{ddl}$, à $P<0,30$ et $43 \%$ sur $D-27$ contre $57 \%$ sur $D-23$ pour les bourdons, $\chi^{2}=1,16$ à $1 \mathrm{ddl}<$ à $P=$ $0,20)$. Mais sur 183 mesures effectuées dont 123 avec abeilles et 60 avec bourdons, les fréquences de passages intralignées ont été très supérieures aux fréquences de passages interlignées (de 1,9 fois plus pour les bourdons à 2,5 pour les abeilles, $\chi^{2}=19$ à $3 \mathrm{ddl}$, $>$ à $P=0,001$ pour les bourdons et $\chi^{2}=27$ à $3 \mathrm{ddl},>$ à $P$ $=0,001$ pour les abeilles).

\section{Les coulures}

\section{Coulures des noeuds reproducteurs}

Le tableau I montre que le nombre de nœuds reproducteurs diminue entre la floraison et la maturité des gousses. Les diminutions de D-23 sont significativement $(P=0,05)$ plus importantes et les taux de coulure apparaissent plus élevés, que ceux de D-27 (de l'ordre de 1,4 à 2,4 fois plus) sauf pour le traitement "abeilles sous cages". Avec D-27, les diminutions sont significatives $(P=0,05)$ en autopollinisation et pollinisation manuelle.

En absence de pollinisateurs (autopollinisation ou déclenchement manuel), les 
Tableau I. Nombre de nœuds reproducteurs au stade "fleurs" puis au stade "gousses mûres" et taux de coulure totale des nœuds reproducteurs des lignées $\mathrm{D}-27$ et $\mathrm{D}-23$ en fonction des conditions de pollinisation.

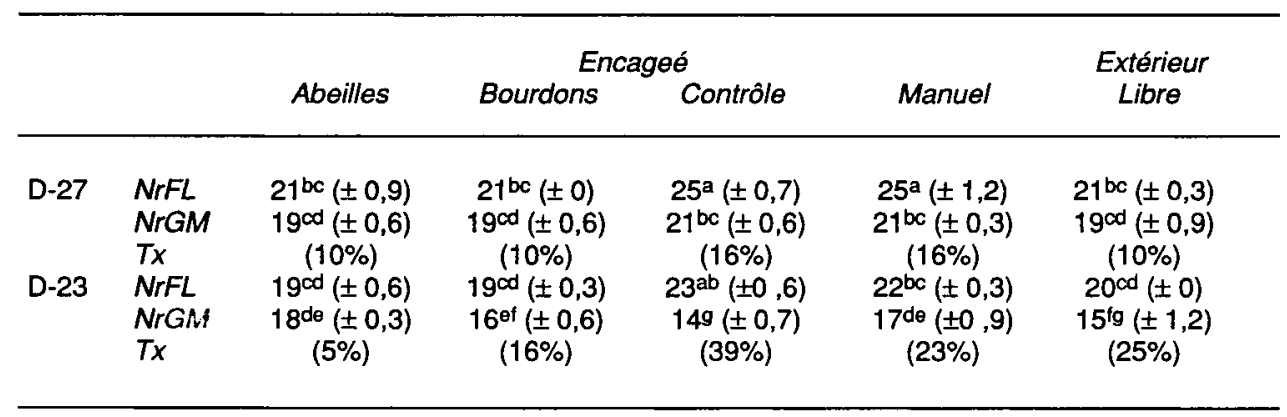

Moyennes ( \pm erreur type). NrFL : nombre de nœuds reproducteurs au stade «fleurs"; NrGM : nombre de nœuds reproducteurs au stade “gousses mûres". Tx : Taux de coulure totale des nceuds reproducteurs (en \%). Les moyennes affectées d'une même lettre ne sont pas significativement différentes.

pourcentages de coulure sont très supérieurs chez D-23 à ce qu'ils sont chez D27. La coulure maximale s'observe chez D-23 en autopollinisation (39\%) et la cou- lure minimale chez D-23 avec abeilles $(5 \%)$. Les écarts entre traitements sont peu prononcés chez D-27 (entre 10 et $16 \%$ ) et forts chez D-23 (entre 5 et $39 \%$ ).
(0)

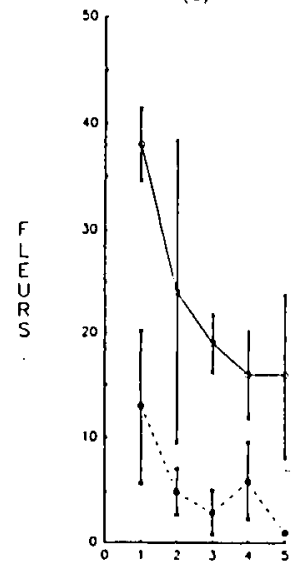

(b)

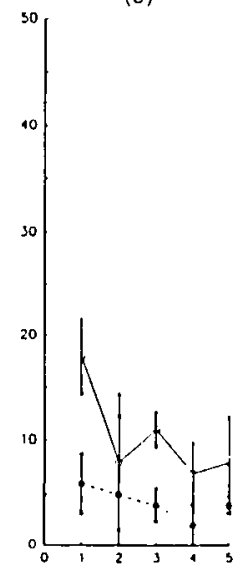

(c)

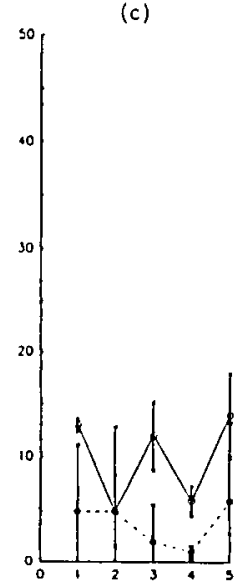

Fig 1. Pourcentages des coulures par secteurs de la plante au stade "fleurs" des lignées D-27 et D23 en fonction des différentes conditions de pollinisation. En abscisse : Traitements : $1:$ encagé avec abeilles; 2 : encagé avec bourdons; 3 : encagé autopoltinisation; 4 : encagé déclenchement manuel; 5 : extérieur pollinisation libre. En ordonnée : Pourcentages des coułures, - - : lignée D-27; - - -.- - -: lignée D-23. (a) secteur 1, nœuds 1 à 5; (b) secteur 2, nœuds 6 à 10; (c) secteur 3, nœuds 11 à 15. 

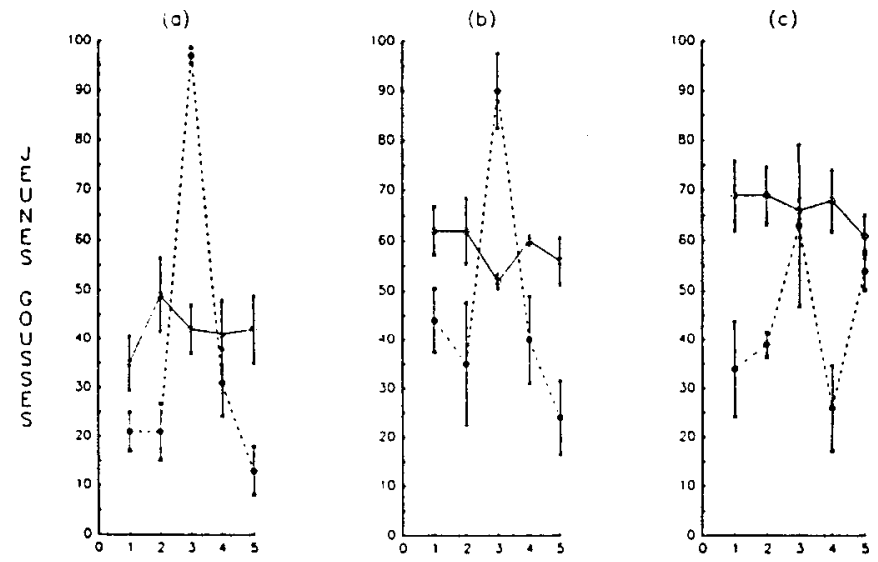

Fig 2. Pourcentages des coulures par secteurs de la plante au stade "jeunes gousses" des lignées D-27 et D-23 en fonction des différentes conditions de pollinisation. Même légende que figure 1.

\section{Coulures relatives par stade phénologique}

\section{Par secteurs de plante}

Les figures 1 à 3 mettent en évidence des coulures plus ou moins importantes qui apparaissent sur différentes parties de la plante à chaque stade phénologique. Les plus élevées se situent pour les fleurs, dans les parties basses des plantes (secteur 1, nœuds 1-5) chez D-27 principalement, de façon plus aléatoire chez D-23 et au contraire, dans les parties les plus hautes (secteur 3, nœuds 11-15) pour les jeunes gousses et les gousses âgées. En autopollinisation, D-23 se signale par une coulure de jeunes gousses et de gousses (a)

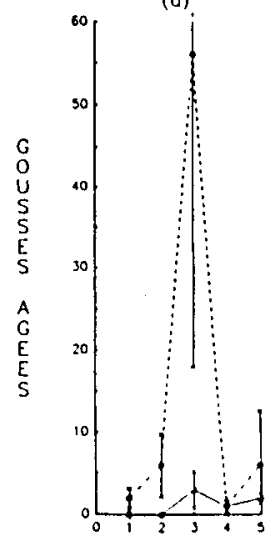

(b)

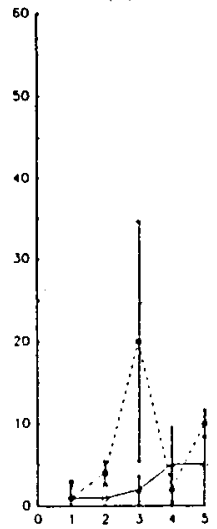

(c)

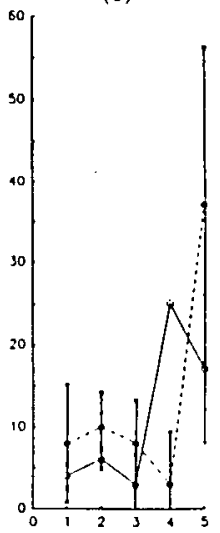

Fig 3. Pourcentages des coulures par secteurs de la plante au stade "gousses âgées" des lignées D-27 et D-23 en fonction des différentes conditions de pollinisation. Même légende que figure 1. 
âgées très élevée dans les parties basses (secteur 1, nœuds 1-5) et intermédiaires des plantes (secteur 2, noeuds 6-10).

Dans l'ensemble, les coulures des fleurs et des jeunes gousses de D-27 sont significativement plus élevées que celles de D$23(P=0,001)$.

Avec les abeilles, les coulures des fleurs apparaissent significativement plus importantes que celles obtenues avec bourdons à tous les niveaux de la plante dans le cas de D-27 et uniquement au niveau bas (secteur 1, nœuds 1-5) dans le cas de D-23.

\section{Par plantes entières}

La figure 4 fait apparaitre des coulures des fleurs relativement faibles au niveau de la plante entière $(9-23 \%$ pour D27 et $3-8 \%$
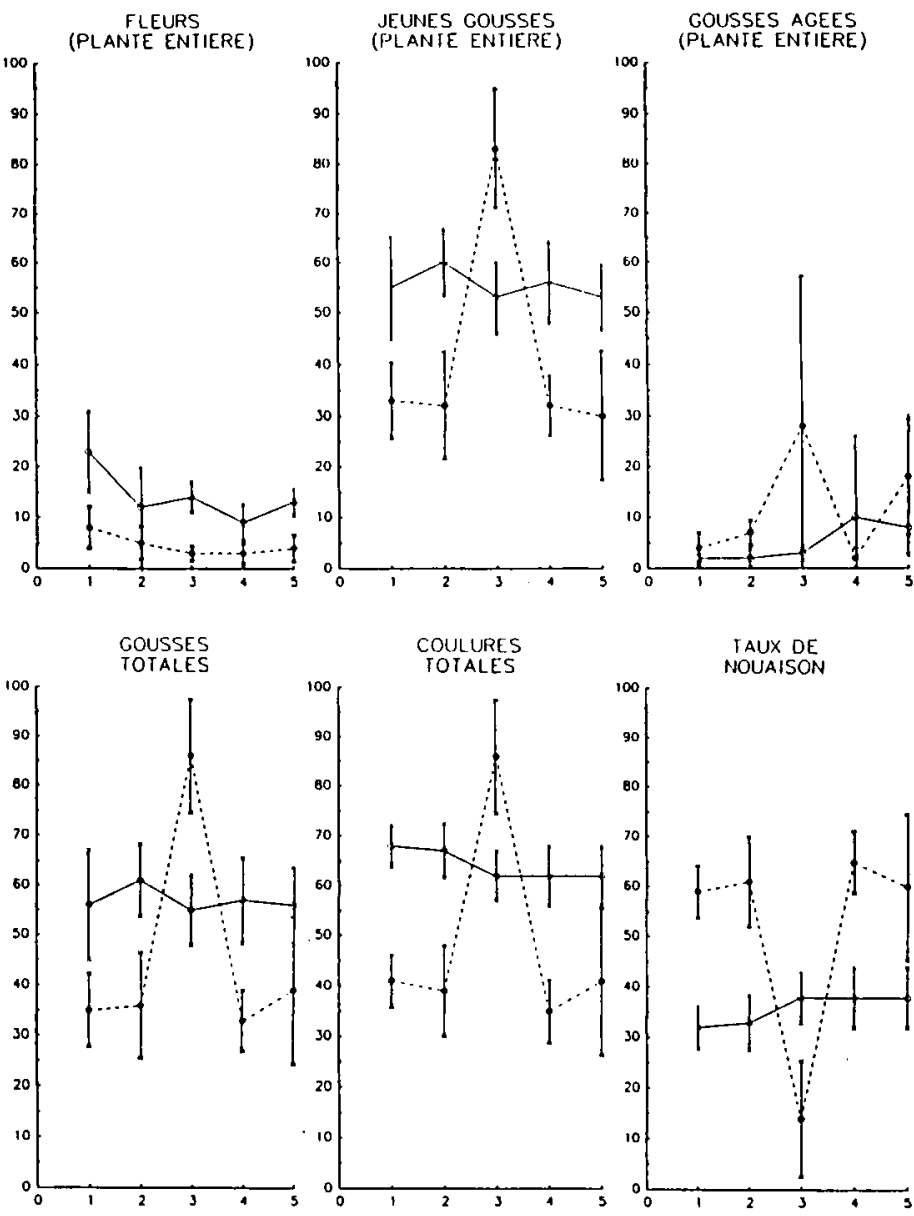

Fig 4. Pourcentages des coulures plantes entières à différents stades phénologiques, des coulures totales et des taux de nouaison en fonction des différentes conditions de pollinisation. Même légende que figure 1. 
pour D-23); celles des jeunes gousses sont nettement plus élevées $(53-60 \%$ pour D-27 et $30-83 \%$ pour D-23). Celles des gousses âgées, au contraire, diminuent fortement $(2-10 \%$ pour $D-27$ et $2-28 \%$ pour D-23).

Avec les abeilles, les taux moyens de coulure des fleurs augmentent par rapport à ceux obtenus avec bourdons notamment (23\% avec abeilles pour $12 \%$ avec bourdons pour $\mathrm{D}-27$, différence significative à $P=0,05$ et $8 \%$ avec abeilles pour $5 \%$ avec bourdons pour D-23, différence non significative à $P=0,05$ ).

En autopollinisation, les taux moyens de coulure des jeunes gousses et des gousses âgées de $D-23(83 \%$ et $28 \%)$ sont significativement $(P=0,001$ et $P=$ $0,05)$ plus élevés comparés aux autres traitements (extrêmes situés entre 30 et $32 \%$ pour les jeunes gousses et entre 2 et $18 \%$ pour les gousses âgées), ce qui ne s'observe pas chez D-27.

Les pourcentages moyens de coulures des gousses âgées de D-27 en autopollinisation $(3 \%)$ sont en effet identiques à ceux obtenus avec les abeilles et les bourdons $(2 \%)$, mais sont inférieurs à ceux qu'on observe avec déclenchement manuel et en pollinisation libre (10 et $8 \%$ différences non significatives à $P=0,05$ ).

Dans l'ensemble, la figure 4 montre aussi que les taux de coulure des fleurs et des jeunes gousses de D-27 pour chacun des traitements apparaissent globalement significativement $(P=0,001)$ plus élevés que ceux de D-23 (de l'ordre de 3 à 5 fois plus pour les fleurs et de l'ordre de 1,8 fois plus pour les jeunes gousses) à l'exception cependant des taux des jeunes gousses obtenus en autopollinisation. Inversement pour les gousses âgées, ce sont ceux de D-23 qui présentent une augmentation, en général, par rapport à ceux de $D-27$, les différences n'étant significatives $(P=0,10)$ qu'en autopollinisation.

\section{Coulures des gousses totales et coulures totales}

La figure 4 montre que les coulures des gousses totales se situent entre 55 et $61 \%$ pour D-27, entre 33 et $86 \%$ pour D-23 et les coulures totales se situent entre 62 et $68 \%$ pour D-27 (soit un taux de nouaison de l'ordre de 32 à $38 \%$ ) et entre 35 et $86 \%$ pour D-23 (soit un taux de nouaison de l'ordre de 14 à 65\%).

En autopollinisation, les coulures totales de D-27 ne sont pas significativement différentes entre traitements $(62 \%$ contre 62 à $68 \%$ ). Par contre, les coulures totales de D-23 apparaissent significativement plus élevées $(P=0,001)$ que celles des autres traitements $(86 \%$ contre 35 à $41 \%$ soit de l'ordre de 2,1 à 2,5 fois plus). Son taux de nouaison, significativement $(P=0,001)$ plus faible que ceux obtenus avec abeilles, bourdons, déclenchement manuel et pollinisation libre (14\% contre $59-65 \%$ ), correspond cependant à $23 \%$ de ceux de ces derniers.

\section{Influence de la pollinisation sur les composantes du rendement}

Les résultats regroupés dans le tableau II permettent de faire un certain nombre de remarques.

D-23 en autopollinisation se distingue significativement $(P=0,001)$ des autres traitements de la même lignée et de D-27 par un nombre inférieur de graines par gousse (2,25 contre $2,77-3,38)$; un accroissement du poids de 1000 graines $(658 \mathrm{~g}$ contre $387-457 \mathrm{~g}$ ); une très forte réduction du rendement $(5,7 \mathrm{~g}$ par plante contre $30,2-41,1 \mathrm{~g})$ et une production de graines plus faible $(8,6$ contre $69,4-94,4)$.

Le tableau II fait apparaître aussi des différences, non significatives du point de vue statistique, indiquant néanmoins les tendances suivantes : 
Tableau II. Composantes du rendement et taux de croisements des lignées D-27 et D-23 en fonction des conditions de pollinisation.

\begin{tabular}{|c|c|c|c|c|c|c|}
\hline & & Abeilles & $\begin{array}{l}\text { Encagée } \\
\text { Bourdons }\end{array}$ & Contrôle & Manuel & $\begin{array}{l}\text { Extérieur } \\
\text { Libre }\end{array}$ \\
\hline \multirow[t]{2}{*}{ NGG } & D-27 & $3,33^{a b}( \pm 0,04)$ & $3,28^{\mathrm{ab}}( \pm 0,1)$ & $3,08^{a b c}( \pm 0,09)$ & $3,38^{a}( \pm 0,10)$ & $2,97^{b c}( \pm 0,19)$ \\
\hline & $D-23$ & $2,83^{c}( \pm 0,16)$ & $3,12^{a b c}( \pm 0,09)$ & $2,25^{d}( \pm 0,11)$ & $2,77^{c}( \pm 0,23)$ & $3,01^{a b c}( \pm 0,20)$ \\
\hline \multirow[t]{2}{*}{ PMG } & $D-27$ & $429 b c d( \pm 22)$ & $416^{\text {bod }}( \pm 18)$ & $441^{b c}( \pm 16)$ & $436^{\text {bod }}( \pm 15)$ & $40 t^{\mathrm{cd}}( \pm 25)$ \\
\hline & $D-23$ & $449 b c( \pm 7)$ & $418^{\mathrm{bcd}}( \pm 11)$ & $658^{a}( \pm 12)$ & $457^{b}( \pm 15)$ & $387^{d}( \pm 29)$ \\
\hline \multirow[t]{2}{*}{ R } & D-27 & $31,6^{\mathrm{a}}( \pm 4,2)$ & $33,3^{a}( \pm 4,9)$ & $39,9^{\mathrm{a}}( \pm 3,5)$ & $41,1^{\mathrm{a}}( \pm 2,7)$ & $31,6^{a}( \pm 0,2)$ \\
\hline & D-23 & 31, fa $_{( \pm 2,8)}$ & $33,5^{a}( \pm 2,3)$ & $5,7^{b}( \pm 0,2)$ & $32,7^{a}( \pm 2,1)$ & $30,2^{a}( \pm 2,4)$ \\
\hline \multirow[t]{2}{*}{ NGP } & D-27 & $73,3^{\mathrm{abc}}( \pm 6,5)$ & $80,0^{\mathrm{abc}}( \pm 10,8)$ & $90,9^{\mathrm{ab}}( \pm 9,4)$ & $94,4^{a}( \pm 5,7)$ & $79,5^{a b c}( \pm 5,4)$ \\
\hline & D-23 & $69,4^{c}( \pm 6,3)$ & $80,2^{a b c}( \pm 5,7)$ & $8,6^{d}( \pm 0,3)$ & $71,9 b c( \pm 6,0)$ & $78,2^{a b c}( \pm 4,7)$ \\
\hline $\mathrm{Ti}$ & $0-27$ & $9,4^{a}( \pm 0,6)$ & $11,6^{\mathrm{a}}( \pm 0,8)$ & 0 & 0 & $5,1^{\mathrm{b}}( \pm 0,5)$ \\
\hline
\end{tabular}

Moyennes ( \pm erreur type). NGG: nombre de graines par gousse; $P M G$ : poids de 1000 graines (en g); $R$ : poids de graines par plante (en g) ou rendement par plante; NGP : nombre de graines par plante; $T I$ : taux de croisements.

- D-27 et D-23 avec bourdons se distinguent des abeilles par un poids de 1000 graines inférieur ( $416 \mathrm{~g}$ contre 429 pour $\mathrm{D}$ 27 et 418 contre 449 pour D-23); un rendement supérieur $(33,3$ contre 31,6 , pour D27 et 33,5 contre 31,1 pour $D-23$ ) et un nombre de graines par plante plus élevé ( 80 contre 73,3 pour D-27 et 80,2 contre $69,4$ pour $D-23)$.

- D-27 en autopollinisation se distingue des abeilles et des bourdons par des moyennes plus faibles (nombre de graines par gousse : 3.08 contre 3.33 et 3.28 ou supérieures (poids de 1000 graines : 441 g contre 429 et 416 , rendements : $39,9 \mathrm{~g}$ contre 31,6 et 33,3 et nombre de graines par plante : 90,9 contre 73,3 et 80,0 ).

\section{Influence de la pollinisation sur les taux de croisements}

Les croisements dans la descendance de D-27 (tableau II) ont été observés dans les cages avec abeilles et avec bourdons ainsi qu'en pollinisation libre.

Les taux de croisements obtenus apparaissent, en moyenne, très faibles $(5,1$ à
$11,6 \%$ ). Ceux des cages avec abeilles diffèrent d'ailleurs peu de ceux des bourdons $(9,4$ et $11,6 \%)$ mais sont significativement plus élevés $(P=0,01)$ que ceux obtenus en pollinisation libre. Ces résultats mettent en évidence un effet combiné cage et insectes qui semble se manifester principalement au niveau des croisements.

\section{DISCUSSION}

Le comportement de butinage des abeilles et des bourdons a été dans l'ensemble satisfaisant. Toutefois, les abeilles ont été beaucoup plus nombreuses dans les cages du fait de la présence de ruchettes très populeuses et elles ont eu tendance à suivre les alignements au cours de leur cheminement comme le montrent les passages intralignées nettement plus fréquents que les passages interlignées.

Les données sur les coulures permettent d'abord de confirmer l'existence chez la féverole de la succession de coulure des organes reproducteurs, depuis l'initiation des boutons floraux jusqu'à maturité 
des gousses, comme cela a déjà été démontré sur cette plante par de nombreux auteurs (Raphalen, 1984) ou sur d'autres espèces de Fabacées (Delaude et Tasei, 1972; Delaude, 1973). Cependant avec les 2 lignées utilisées d'importantes variations sont apparues.

La lignée qui s'autopollinise (D-27) s'est en effet montrée plus sensible aux coulures des fleurs et des gousses que la lignée à déclenchement obligatoire (D-23) à l'exception cependant des coulures de fleurs et des gousses de D-23 en autopollinisation.

En raison de sa sensibilité plus forte aux coulures, la lignée autofertile (D-27) aurait pu montrer une baisse des rendements à la récolte. Or ils ont été, au contraire, équivalents à ceux de la lignée D-23. La pollinisation entomophile n'est pas en cause dans ce cas, puisque les rendements de la lignée autofertile obtenus en absence d'insectes ont été peu différents de ceux obtenus en leur présence. Nous pensons qu'il s'agirait plutôt d'un phénomène de compensation ayant entraîné un certain ajustement entre floraison et production de graines chez la lignée autopollinisée. En effet, après une forte coulure des fleurs, la production de graines est plus élevée chez cette lignée que chez la lignée non autofertile.

Si l'on examine les coulures significativement plus importantes des premières fleurs situées dans les parties basses des plantes (nceuds 1 à 5), 2 interprétations sont possibles, selon que l'on considère celles qui se sont produites dans les cages avec abeilles ou celles qui ont été observées dans chacune des conditions de pollinisation avec la lignée $D-27$ seulement.

Les coulures importantes des premières fleurs qui se sont produites dans les cages avec abeilles sur les 2 lignées de féverole pourraient s'expliquer par une anomalie due au surbutinage des abeilles trop nombreuses pour les fleurs offertes. Un meilleur équilibre semble s'établir ensuite entre le nombre de fleurs exploitables qui augmente progressivement sur chaque plante au fur et à mesure de leur développement pour pallier, dans une certaine mesure, la réponse fournie par les butineuses. Les pourcentages plus faibles des coulures des fleurs obtenus sur les parties moyennes et supérieures des plantes de la lignée D-23 principalement, semblent le démontrer.

Le passage trop souvent répété des abeilles sur les fleurs aurait provoqué, soit un assèchement du stigmate, ne permettant plus l'hydratation du pollen par les sécrétions stigmatiques et sa germination (Dumas, 1984), soit une accumulation excessive de leurs sécrétions salivaires dont on connait le pouvoir d'inhibition de la germination du pollen (Singh et Boynton, 1949; Johansen, 1955; Lavie, 1960; Maurizio, 1960; Mesquida et Renard, 1989). Mais, il s'agit d'hypothèses qu'il serait d'un grand intérêt de vérifier par la suite. On peut, d'autre part, penser que le rapport (fleurs/organes photosynthétiques), faible dans les premiers stades s'accroît par la suite et permettrait une meilleure alimentation d'organes reproducteurs (fleurs et jeunes gousses).

Bien que cette étude soit insuffisamment avancée pour donner une explication correcte au phénomène, il semble que la surexploitation des fleurs par les abeilles entraîne une diminution de leur efficacité pollinisatrice. Celle-ci peut être rapprochée des observations de Pritsch (1970); Holm (1972) et Bogoyavlenskii (1970) en conditions de plein air. Dans nos conditions d'expérimentation l'effet cage pourrait amplifier le phénomène. 
Les coulures des premières fleurs qui ont été plus élevées dans chacune des conditions de pollinisation avec la lignée qui s'autopollinise (D-27) par rapport à D23 semblent liées davantage à sa plus forte sensibilité aux coulures que nous avons signalée précédemment.

D'une manière générale, le faible nombre de jeunes gousses (de $14 \%$ à $65 \%$ dans nos conditions d'expérimentation) qui arrivent à maturité comme cela a déjà été signalé par Tasei (1984) sur féverole et Delaude et Tasei (1972) sur luzerne, se trouve confirmé.

Mais les variations observées en fonction des secteurs de plante, montrent que, contrairement au cas des fleurs, les jeunes gousses situées dans les parties basses sont moins prédisposées aux coulures que celles situées dans les parties hautes. Les jeunes gousses qui apparaissent d'abord à la base de la plante se développent rapidement, elles sont mieux nourries, les grains grossissent plus vite et coulent peu. Les potentialités de production de gousses semblent ainsi largement atteintes à ce niveau de la plante. Les suivantes, plus hautes coulent plus selon un processus d'autorégulation de la plante elle-même qui semblerait intermédiaire à celui que l'on observe habituellement chez les féveroles à dominance apicale réduite (Berthelem, communication personnelle).

L'absence de pollinisation entomophile joue un rôle évident chez la lignée D-23 à déclenchement obligatoire en autopollinisation.

Compte tenu des taux de nouaison et des composantes du rendement observés, nous pouvons dire que l'influence de la pollinisation entomophile s'est avérée positive et significative sur la lignée D-23 et sans effet sur la lignée D-27.

En effet, chez la lignée D-23, l'absence de déclenchement par les insectes a été marquée par une forte diminution des taux de nouaison et des composantes du rendement. Cependant, elle a présenté, en conditions d'autopollinisation, un taux de nouaison non négligeable $(23 \%$ de ceux obtenus en pollinisation entomophile). Cette production de gousses pourrait correspondre à de l'autopollinisation spontanée ou à un phénomène d'autodéclenchement mécanique suivi d'autofécondation (secouement accidentel ou par le vent). Cela peut correspondre aussi à une augmentation naturelle de l'autofertilité en relation avec l'âge de la plante comme le montre la réduction des coulures des étages hauts par rapport au bas chez D23.

Le taux très faible de croisements observé dans cette expérience par rapport au taux moyen d'allogamie de la féverole (40$50 \%$, Bonjean et Helle, 1979) appelle 2 commentaires.

- ce taux a été mesuré sur une lignée qui s'autopollinise largement (lignée D-27), ce qui pourrait indiquer que l'autofertilité s'accompagnerait d'une sélection gamétique pour l'autopollen par rapport à l'allopollen, d'où faible proportion d'“hybridation" ou bien de la précocité de la fécondation avec l'autopollen, rendant sans effet l'apport d'allopollen.

- en revanche, si ce taux très faible d'intercroisements devait être la règle générale chez la féverole, indépendamment du degré d'autopollinisation de la lignée, cela pourrait avoir une répercussion importante sur les stratégies de sélection dans cette espèce. L'analyse des croisements réciproques par une méthode autre que celle des marqueurs tégumentaires (marqueurs enzymatiques) réalisée par Tasei et Carre, devrait nous indiquer si le taux de croisement est aussi faible pour une lignée (D23) non autopollinisée.

II resterait à discuter l'intérêt pratique de cette étude en sélection : 
- Si la présence d'insectes pollinisateurs ne permet de modifier ni les coulures ni la productivité des lignées autopollinisées du type D-27 et si ces dernières sont seules capables de s'autoréguler et de s'ajuster naturellement aux diverses conditions de milieu par des phénomènes de compensation, it serait souhaitable de donner en sélection la préférence aux lignées peu nectarifères, moins attractives vis-à-vis des insectes (transformation de la biologie florale vers l'autopollinisation). Cette transformation permettrait de lever les contraintes techniques inhérentes à la structure allogame de la féverole (cages d'isolement, isolements individuels etc).

- Au contraire, dans l'optique de la création de variétés hybrides ou synthétiques, l'allogamie étant nécessaire, il serait souhaitable, avec les lignées non autopollinisées du type D-23 de sélectionner les plus nectarifères de manière à les rendre plus attractives vis-à-vis des insectes pollinisateurs et augmenter ainsi les fréquences de la pollinisation croisée. Mais pour favoriser l'allogamie, il faudrait aussi tenir compte du comportement de butinage des insectes pollinisateurs et par conséquent du dispositif à appliquer. Or, dans notre expérimentation, notre dispositif, constitué, de l'alternance de 2 bandes de 2 lignes chacune par lignée, s'est montré peu favorable aux croisements entre lignées. Un dispositif en plantes alternées aurait peutêtre permis d'améliorer leur importance.

\section{CONCLUSION}

Cette étude a permis de montrer que l'influence de la pollinisation entomophile sur les taux de coulure et la productivité de la féverole peut-être nulle ou très positive selon la biologie reproductive et l'origine de la lignée.
Elle a été sans effets apparents sur la lignée autopollinisée (D-27). L'autopollinisation appraît prépondérante chez ce génotype qui dispose en outre, en dépit de sa sensibilité aux coulures, de systèmes de compensation qui lui confèrent de grandes possibilités d'ajustements de sa productivité, quant aux conditions de pollinisation.

L'influence de la pollinisation entomophile a été par contre très positive sur la lignée non autopollinisée (D-23). Elle s'est manifestée par une diminution des taux de coulures absolues et une augmentation des nouaisons et des rendements. Le déclenchement de la colonne staminale joue un rôle important sur ce génotype et la présence d'insectes (abeilles ou bourdons) apparait nécessaire pour assurer efficacement sa pollinisation. Cette lignée présente cependant une aptitude non négligeable à l'autopollinisation.

\section{REMERCIEMENTS}

Nous tenons à remercier tout particulièrement Messieurs Duc (INRA de Dijon) pour la fourniture de semences des lignées de féveroles utilisées dans cette étude et F Brunet (Lycée Agricole d'Arras) pour les longues observations qu'il a effectuées tout au long de son stage.

Summary - Effect of pollination treatments on reproductive organ abortion rates, yield and crossing rates in 2 lines of spring faba bean (Vicia faba $L$ var equina Steudel). The effects of 5 pollination treatments on reproductive organ abortion rates, fruiting rate, yield components and crossing were studied in a self-pollinating (D-27) and a non selfpollinating (D-23) line of faba bean. The experimental design consisted of a completely randomized block with 3 replications, 5 treatments and 2 varieties per treatment. Pollination treatments were as 
follows: 1) cages with honeybees (Apis mellifera L) (a small hive consisting of 5 frames of honeybees per cage), 2) cages with bumblebees ( 2 queens of Bombus lapidarius $L$ per cage), 3) cages without insects (self-pollination), 4) cages without insects but hand pollinated (= tripped), 5) outside standard open pollination. Cages used in this experiment were $3 \times 3 \times 2 \mathrm{~m}$ $(\mathrm{L} \times \mathrm{W} \times \mathrm{H})$ and made of 2-mm mesh cloth. Two rows of 10 plants for each of the 2 lines were sown in each cage. Observations were conducted on insect foraging behaviour, abortion rate of reproductive organs (nodes, flowers and pods) for 5 plants per line, per treatment and per block.

In all cages with honeybees, flower abortions at the bottom of the plants were significantly higher than at the top $(38 \%$ and $13 \%$ at the bottom versus $13 \%$ and $5 \%$ at the top for D-27 lines and D-23 line respectively), indicating possible "over visitation" due to overpopulation (bees/plant) of honeybees per cage. Line D-27 showed a greater susceptibility to flower abortion than line D-23.

When compared to selfing, entomophilous pollination (honeybees or bumblebees) had no significant effect on flower abortion, pod set and yield components for line D-27. Self-pollination was thus considered predominant in this genotype. This genotype also seemed to possess good capacities for compensation when exposed to various environmental conditions.

In contrast, entomophilous pollination appeared necessary for line D-23. In the absence of bees, the abortion rate of yound pods ( $<2 \mathrm{~cm}$ long) increased significantly ( $83 \%$ vs $33 \%$ with honeybees and $32 \%$ with bumblebees, $P=0.001$ ). The same was also observed for older pods (>2 cm long; $28 \%$ vs 4 and $7 \%, P=0.01$ but less than $4 \mathrm{~cm}$ long). Absolute abortion rate was higher under selfing conditions when compared to insect pollination ( $86 \%$ vs 41 and $39 \%, P=0.001)$. With selfpollination there was a significant restriction of the pod setting rate ( 8.6 vs 69.4 and $80.2, P=0.001$ ), the number of seeds per pod (2.25 vs 2.83 and $3.12, P=0.001)$ and the yield (5.7 vs 31.1 and $33.5, P=0.001)$. Nevertheless, fructification for this line under self-pollination (ie without insects or tripping) was $23 \%$ of that with insects. The outcrossing rate of line $D-27$ by line $D-23$ was $\approx 10 \%$ in treatments 1 or 2 and $5 \%$ in treatment 5 .

\section{Apis mellifera / Bombus / pollination / organ abortion / Vicia faba}

\section{Zusammenfassung - Bestäubung zweier Linien der Frühjahrs-} Pferdebohne (Vicia faba $L$ var equina Steudel). Auswirkungen auf den Abfall der Blütenorgane, die Produktivität und die Kreuzungsrate. Der Einflu $B$ von fünf verschiedenen Bestäubungs-Bedingungen auf den Abfall der Blütenorgane, den Fruchtansatz, die Ertragsfaktoren und auf die Kreuzung wurden an einer selbstbestäubenden (D-27) und einer selbststerilen Linie (D-23) der Pferdebohne untersucht. Der Versuchsansatz bestand aus einem zufällig angeodneten Block mit 5 Behandlungen und drei Wiederholungen je $\mathrm{Be}$ handlung. Die Bestäubungsbedingungen waren wie folgt: 1) Käfig mit Honigbienen (Apis mellifera L), und zwar ein kleines Volk mit 5 Rähmchen je Käfig. 2) Käfig mit Hummeln (zwei Königinnen von Bombus lapidarius je Käfig). 3) Käfig ohne Insekten (Selbstbestäubung). 4) Käfig ohne Insekten, aber mit Handbestäubung ("trippen"). 5) Freie Bestäubung ohne Käfig. Die in diesem Versuch benutzten Käfige waren 3 $\times 3 \times 2 \mathrm{~m}(\mathrm{~L} \times \mathrm{B} \times \mathrm{H})$ groß und aus $2 \mathrm{~mm}$ Netzgewebe gefertigt. In jeden Käfig wurden zwei Reihen zu je 10 Pflanzen von jeder der beiden Linien ausgesät. Es wur- 
den Beobachtungen über das Sammelverhalten der Insekten, die Abfallrate der Blütenorgane (Blütenstand, Blüte, Schote) von fünf Pflanzen je Linie, je Behandlung und je Block durchgeführt.

In sämtlichen Käfigen mit Honigbienen war der Blütenabfall im unteren Teil der Pflanze signifikant höher als an der Spitze (38\% und $13 \%$ unten gegen $13 \%$ und $5 \%$ and der Spitze fur die beiden Linien D-27 und D-23). Dies könnte als Folge einer "Überbearbeitung" der Blüten wegen des reichlichen Besatzes der Käfige mit Bienen aufgefaßt werden. Die Linie D-27, mit stärkerem Blütenansatz als Linie D-23 $(9,82$ Blüten per Blütenstand bei D-27 gegen 7,08 bei $D-23$ ), hat sich hinsichtlich des Abfalls als empfindlicher gezeigt.

Im Vergleich zur Selbstbestäubung hatte die Insektenbestäubung (Bienen oder Hummeln) bei der Linie D-27 keinen signifikanten Einfluß auf Blütenabfall, Samenansatz und die Ertragskomponenten. Der Gesamtabfall betrug mit Bienen und Hummeln $68 \%$ und $67 \%$, bei Selbstbestäubung $62 \%$, der Ertrag je Staude 73,3 Bohnen gegen 90,9 und an Gewicht 31,9 g gegen $39,9 \mathrm{~g}$. Bei diesem Genotyp kann also die Selbstbestäubung als vorherrschend angesehen werden. Er schien auch gute Kompensationsmöglichkeiten gegenüber verschiedenen Umwelteinflüssen zu besitzen.

Demgegenüber scheint für die Linie $D$ 23 Insektenbestäubung notwendig und obligat zu sein. Bei dieser Linie wurde auch ein erhöhter Abfall der Jungen Schoten (von mindestens $2 \mathrm{~cm}$ Länge) beobachtet: $83 \%$ gegen 33 und $32 \%$ mit Bienen und Hummeln. Ebenso bei älteren Schoten (Länge über $2 \mathrm{~cm}$ ): $28 \%$ gegen 4 und $7 \%$ mit Bienen und Hummel; beim Totalabfall waren die Zahlen $86 \%$ gegen 41 und $39 \%(P=0,001)$. Ferner war bei Selbstbestäubung eine deutliche Senkung des Schotenansatzes ( 8,6 gegen 69,4 und
$80,2)$, der Zahl der Samen je Schote $(2,25$ gegen 2,83 und 3,12 ) und dem Ertrag $(5,7 \mathrm{~g}$ gegen 31,1 und $33,5 \mathrm{~g})$ festzustellen $(P=0,001)$. Die Rate des Fruchtansatzes betrug bei dieser Linie unter den Bedingungen der Selbstbestäubung (dh ohne Insekten oder künstlichem "Tripping") etwa $23 \%$ dessen, was bei Anwesenheit von Insekten zu erwarten war. Die Kreuzungsrate der Linie D-27 war niedrig, 9,4\% mit Bienen und $11,6 \%$ mit Hummeln, und sogar nur $5 \%$ im Freien.

\section{Apis mellifera / Bombus / Bestäubung / Abfall der Blütenorgane / Vicia faba}

\section{RÉFÉRENCES}

Berthelem P (1976) Amélioration génétique de la féverole. Cultivar $89,48-53$

Bogoyavlenskii GS (1975) The multiplicity of visits to sainfoin by honey bees and its importance for the yieid of seeds. CR 3e Symp Int Pollination, Prague, 1974. Bull Tech Apic 2, 121-127

Bonjean A, Helle JL (1979) La féverole. Sa biologie florale. Rev Fr Apic 377, 360-363

Dagnelie $P$ (1973) Théorie et méthodes statistiques. Applications agronomiques. Les Presses Agronomiques de Gembloux, tome I, $378 p$

Delaude A (1973) Influence de quelques facteurs externes sur la coulure des fleurs et la production de graines de luzerne. Zesz Probl Postepow Nauk Rolniczych 131, 33-42

Delaude A, Taseï JN (1972) Premières observations sur la pollinisation et la coulure des fleurs de luzerne en Charentes (Medicago sativa L). Apidologie 3, 79-97

Dumas C (1984) De l'ovule à la graine : bases cytologiques et physiologie de la fécondation. In : Pollinisation et productions végétales ( $P$ Pesson, J Louveaux, eds) INRA, Paris, 47-72

Free JB (1962) The behaviour of honey bees visiting field beans (Vicia faba). J Anim Ecol 31, 497-502 
Free JB (1970) Insect Pollination of Crops. Acad Press, London

Holm SN (1972) Seed yields in red clover in relation to the number of pollinating bees as influenced by a growth regulator. $\mathrm{Kg} / \mathrm{Vet}$ Landbohøjsk Arsskrift, 127-141

Johansen CA (1955) Bee collected pollen for artificial pollination of apples. Am Bee $J$ 95, 352-353

Lavie $P(1960)$ Les substances antibactériennes dans la colonie d'abeilles (Apis mellifica $\mathrm{L}$ ). Ann Abeille 3, 103-183, 201-305

Maurizio A (1960) Pollenkeimung hemmende Stoffe im Körper der Honigbiene. Atti ufficiali, XVlle Congr Int Apic, Imola, 2, 23-25

Mesquida J, Marilleau R, Pham-Delegue MH, Renard M (1988) A study of rapeseed (Brassica napus $L$ var oleifera Metzger) flower nectar secretions. Apidologie 19, 307-318

Mesquida J, Renard M (1989) Etude de l'aptitude à germer in vitro du pollen de colza (Brassica napus $L$ ) récolté par l'abeille domestique (Apis mellifica L). Apidologie 20, 197-205

Poulsen MH (1973) The frequency and foraging behaviour of honeybees and bumble bees on field beans in Danmark. J Apic Res 12 , 75-80

Pouvreau A (1965) Sur une méthode d'élevage des bourdons (Bombus Latr) à partir de reines capturées dans la nature. Ann Abeille 8, 147-159

Pritsch G (1970) Untersuchungen über die Bedeutung der Honigbienen (Apis mellifica $\mathrm{L}$ ) bei ber Sicherung der Samenerträge des Winterwicken (Vicia villosa Roth). Arch Geflügelzucht Kleintierkd 19, 133-153

Raphalen (1984) Compte rendu, essai agrométéo ITCF-SEA. Relations plante/milieu

Singh S, Boynton D (1949) Viability of apple pollen in pollen pellets of honeybees. Proc Am Soc Hortic Sci 53, 148-152

Tasei JN (1976) Les insectes pollinisateurs de la féverole d'hiver (Vicia faba equina $L$ ) et la pollinisation des plantes mâle stérile en production de semence hybride. Apidologie 7, 138

Tasei JN (1984) Légumineuses fourragères et protéagineuses. In : Pollinisation et productions végétales (Pesson $\mathrm{P}$, Louveaux $\mathrm{J}$, eds) INRA, Paris, 261-308 